\title{
A variant of the McKay-Miller-Širáň construction for mixed graphs
}

\author{
Nacho López, Hebert Pérez-Rosés, and Jordi Pujolàs ${ }^{2}$ \\ Department of Mathematics \\ University of Lleida \\ Lleida, Spain
}

Mária Ždímalová ${ }^{3}$

Department of Mathematics and Constructive Geometry

Slovak University of Technology

Bratislava, Slovakia

\begin{abstract}
The Degree/Diameter Problem is an extremal problem in graph theory with applications in network design. One of the main research areas in the Degree/Diameter Problem consists of finding large graphs whose order approach the theoretical upper bounds as much as possible. In the case of directed graphs there exist some families that come close to the theoretical upper bound asymptotically. In the case of undirected graphs there also exist some good constructions for specific values of the parameters involved (degree and/or diameter). One such construction was given by McKay, Miller, and Širáň in [5], which produces large graphs of diameter 2 with the aid of the voltage assignment technique. Here we show how to re-engineer the McKay-Miller-Širáň construction in order to obtain large mixed graphs of diameter 2, i.e. graphs containing both directed arcs and undirected edges.
\end{abstract}

Keywords: Network design, Degree/Diameter Problem, mixed graphs, voltage assignment 


\section{Introduction}

The Degree/Diameter problem is a classic extremal problem in network design. The goal is to investigate the largest order of a graph subject to constraints in its maximum degree and its diameter. In particular, the Degree/Diameter problem for mixed graphs asks for the largest possible number of vertices $n(r, z, k)$ in a mixed graph with maximum undirected degree $r$, maximum directed out-degree $z$ and diameter $k$.

This problem has been extensively studied for purely undirected and directed graphs, but not much is known for mixed graphs. Much of what is known is summarized in [4]. The upper bound for $n(r, z, k)$ (also known as the Moore bound) is given in [2]. In the case of diameter 2, the Moore bound specializes to

$$
M(r, z, 2)=1+z+(r+z)^{2} .
$$

The case of diameter 2 is significant because this is the only case where the upper bound can be reached. Graphs that reach the upper bound are called Moore graphs, and they do not exist for all combinations of $r$ and $z$. In the cases where Moore graphs do not exist, or are not known to exist, we aim at constructing the graph whose order approaches the Moore bound as much as possible.

A construction technique that has proved very successful in undirected graphs is voltage assignment. In its original form, voltage assignment takes a base digraph and a group to obtain a new, larger digraph. By dropping arc directions, or by taking a symmetric generating set for the group, one can also construct undirected graphs.

Let $G$ be a digraph, $\Gamma$ a finite group, and $\alpha: D(G) \rightarrow \Gamma$ a labelling of the arcs with elements of $\Gamma$ (in this context the graph may have loops and (or) parallel edges). The labels are usually called voltages, and $\alpha$ is a voltage assignment.

Given the conditions above, a new graph $G^{\alpha}$ (called the lift) is constructed as follows: The vertex set and the arc set of the lift are $V\left(G^{\alpha}\right)=D(G) \times \Gamma$ and $D\left(G^{\alpha}\right)=D(G) \times \Gamma$ (sometimes is usefull to use the subscripts for the $\Gamma$ coordinates of ordered pairs). Incidence in the lift is defined as follows: For

\footnotetext{
1 The first three authors acknowledge the support of the Spanish Ministry of Economy and Competitiveness, under grant MTM2013-46949-P. Mária Ždímalová was supported by the Slovak Research Grant VEGA 1/0065/13.

2 Email: [nlopez, hebert.perez, jpujolas]@matematica.udl.es

3 Email: zdimalova@math.sk
} 
any $\operatorname{arc} e$ from $u$ to $v$ in $G$ and any $g \in \Gamma$ there is exactly one arc $e_{g}$ in the lift $G^{\alpha}$; this arc emanates from the vertex $u_{g}$ and terminates at the vertex $v_{g \alpha(e)}$.

It is easy to see that the arc $\left(e^{-1}\right)_{g \alpha(e)}$ of the lift $G^{\alpha}$ emanates from $v_{g \alpha(e)}$ and terminates at $u_{g}$, because $\alpha\left(e^{-1}\right)=(\alpha(e))^{-1}$. The pair of $\operatorname{arcs} e_{g}$ and $\left(e^{-1}\right)_{g \alpha(e)}$ constitutes an undirected edge of the lift $G^{\alpha}$; therefore, for the reverse arcs in the lift we have $\left(e_{g}\right)^{-1}=\left(e^{-1}\right)_{g \alpha(e)}$. In particular, any Cayley graph is a lift of a bouquet graph (a graph with a single vertex and self-loops).

Many properties of the lift can be identified by examining walks in the base graph. A walk of length $m$ in a graph $G$ is a sequence $W=e_{1} e_{2} \ldots e_{m}$ where $e_{1}$ are arcs of $G$, such that the terminal vertex of $e_{i-1}$ is the same as the initial vertex of $e_{i}, 2 \leq i \leq m$. We say that $W$ is a $u-v$ walk if $u$ is the initial vertex of $e_{i}$ and $v_{i}$ is the terminal vertex of $e_{m}$. If $u=m$ then the walk $W$ is said to be closed, or closed at $u$. If $\alpha$ is a voltage assignment on $G$, then the net voltage of $W$ is defined as the product $\alpha(W)=\alpha\left(e_{1}\right) \alpha\left(e_{2}\right) \ldots \alpha\left(e_{m}\right)$. For more details in relation to the voltage assignment technique see [3].

In order to determine the diameter of the lift, we will make use of the following result:

Lemma 1.1 ([1]) Let $\alpha$ be a voltage assignment on a graph $G$ in a group $\Gamma$. Then, the diameter of $G^{\alpha}$ is at most $k$ if, and only if, for each ordered pair of vertices $u, v$ of $G$ (possibly with $u=v$ ), and for each $g \in \Gamma$, there exists a $u-v$ walk of length $\leq k$ of net voltage $g$.

\section{The McKay-Miller-Širáň construction}

In [5] McKay, Miller and Širáň describe a family of large vertex-transitive graphs with degree $(3 q-1) / 2$, where $q$ is a prime power congruent with 1 (mod 4 ), though they contend that the construction generalizes to all prime powers. The base graphs used in [5] are complete bipartite graphs with loops. Later, Šiagiová showed that the McKay-Miller-Širáň graphs can also be obtained as lifts of dipoles [6]. The McKay-Miller-Širáň graphs still stand as the best family of graphs of diameter 2, asymptotically.

We now focus on Jana Šiagiová's reformulation of the McKay-Miller-Širán construction. As we mentioned before, the base graph is a dipole, i.e. a directed pseudoraph with two vertices, $u$ and $v$, and multiple arcs from $u$ to $v$, as well as multiple loops at each vertex. Voltages are taken from $F_{q}^{+} \times$ $F_{q}^{+}$, where $F_{q}^{+}$denotes the additive group of the finite field $\mathbb{F}_{q}$, where $q \equiv 1$ $(\bmod 4)$. The dipole has exactly $(q-1) / 4$ loops at each vertex, and $q$ arcs from $u$ to $v$. Let $\xi$ be a primitive root of $\mathbb{F}_{q}$. The voltages are defined as 
follows:

(i) Every arc emanating from $u$ to $v$ has voltage $\left(g, g^{2}\right)$, for all $g \in F_{q}^{+}$.

(ii) Loops at vertex $u$ have voltage $\left(0, \xi^{2 i+1}\right)$, for all $0 \leq i<\frac{q-1}{4}$.

(iii) Loops at vertex $v$ have voltage $\left(0, \xi^{2 i}\right)$, for all $0 \leq i<\frac{q-1}{4}$.

The lift defined by the above voltage assignment is a directed graph. It is proved in [6] that, if the orientation of the arcs is removed, we obtain a vertex-transitive graph of degree $\frac{3 q-1}{2}$ and diameter 2 .

\section{Our variant}

Next we proceed to give a construction of a family of mixed graphs with order approaching the Moore bound. This construction is a variant of the one given by Jana Šiagiová. Again, let $\mathbb{F}_{q}$ denote a finite field of prime power order $q \equiv 1(\bmod 4)$. The base group is $F_{q}^{+} \times F_{q}^{+}$, where $F_{q}^{+}$denotes the additive group of $\mathbb{F}_{q}$. Now, our base graph is the dipole $D_{q}^{*}$ on two vertices $u$ and $v$, containing $q$ arcs both from $u$ to $v$ and from $v$ to $u$, and $\frac{q-1}{2}$ loops at each vertex. Let $\xi$ be again a primitive root of $\mathbb{F}_{q}$. The voltages $\alpha_{1}$ over $D_{q}^{*}$ are defined as follows:

(i) Every arc emanating from $u$ to $v$ has voltage $\left(g, g^{2}\right)$, for all $g \in F_{q}^{+}$.

(ii) Every arc emanating from $v$ to $u$ has voltage $\left(g,-g^{2}-1\right)$, for all $g \in F_{q}^{+}$.

(iii) Loops at vertex $u$ have voltage $\left(0, \xi^{2 i+1}\right)$, for all $0 \leq i<\frac{q-1}{2}$.

(iv) Loops at vertex $v$ have voltage $\left(0, \xi^{2 i}\right)$, for all $0 \leq i<\frac{q-1}{2}$.

Theorem 3.1 For any prime power $q \equiv 1(\bmod 4)$ the lift $\left(D_{q}^{*}\right)^{\alpha_{1}}$ is a mixed graph on $2 q^{2}$ vertices of diameter 2 , undirected degree $r=\frac{q-1}{2}$ and directed out-degree $z=q$.

Proof: Since $\left|F_{q}^{+} \times F_{q}^{+}\right|=q^{2}$ and $D_{q}^{*}$ has two vertices, the lift graph $\left(D_{q}^{*}\right)^{\alpha_{1}}$ has order $2 q^{2}$. To see that $r=\frac{q-1}{2}$ and $z=q$ we observe that none of the voltages defined on the $q$ arcs from $u$ to $v$ have an inverse in a voltage defined on an arc from $v$ to $u$. Indeed, otherwise we would have $\left(g, g^{2}\right)+$ $\left(h,-h^{2}-1\right)=(0,0)$, for some $g, h \in F_{q}^{+}$, and this is impossible. Besides, the sets $X_{1}=\left\{\xi^{2 i+1} \mid 0 \leq i<\frac{q-1}{2}\right\}$ and $X_{2}=\left\{\xi^{2 i} \mid 0 \leq i<\frac{q-1}{2}\right\}$ have the property that $X_{1} \cup X_{2}=F_{q}^{+} \backslash\{0\}, X_{1} \cap X_{2}=\emptyset$ and $($ since $q \equiv 1(\bmod 4))$ they are respectively closed under additive inverses. In fact, $X_{1}=\left\{\xi^{2 i+1},-\xi^{2 i+1} \mid 0 \leq\right.$ $\left.i<\frac{q-1}{4}\right\}$ and $X_{2}=\left\{\xi^{2 i},-\xi^{2 i} \mid 0 \leq i<\frac{q-1}{4}\right\}$. This means that for any loop emanating from $u$ (or $v$ ), there is another one with the inverse voltage, that 
is, both generate two closed walks of length 2 and net voltage $(0,0)$ in $\left(D_{q}^{*}\right)^{\alpha_{1}}$. Hence $r=\frac{q-1}{2}$ and $z=q$. To prove that $\operatorname{diam}\left(\left(D_{q}^{*}\right)^{\alpha_{1}}\right)=2$ we only have to check that for any pair of vertices $u$ and $v$ (not necessary distinct) of $D_{q}^{*}$ there exists at least one walk $W$ of length $\leq 2$ with net voltage $(g, h)$, for every $(g, h) \in F_{q}^{+} \times F_{q}^{+}$.

(i) Walks $W$ from $u$ to $u$ with net voltage $(g, h)$ : If $(g, h)=(0,0)$ the walk $W$ consists of two loops with voltages $\left(0, \xi^{2 i+1}\right)$ and $\left(0,-\xi^{2 i+1}\right)$ for any $0 \leq i<\frac{q-1}{4}$. If $g=0$ and $h \in X_{1}$, then the walk $W$ consists of a single loop with voltage $(0, h)$. If $h \notin X_{1}, h \neq 0$, then also $-h \notin X_{1}$ and as $0 \notin X_{1}$ and $h \notin h+X_{1}$, we see that $X_{1}$ and $h+X_{1}$ have a nonempty intersection: Indeed, suposse that $X_{1} \cap\left(h+X_{1}\right)=\emptyset$, then since $\left|h+X_{1}\right|=\left|X_{1}\right|=\frac{q-1}{2}$, we derive that $\left|X_{1}\right|+\left|h+X_{1}\right|=q-1$, but this is impossible since $0 \notin X_{1}$ and $h \notin X_{1}$. Hence, there exist $x, y \in X_{1}$ such that $x=h+y$. In this case $W$ is composed of two loops with voltages $(0, x)$ and $(0,-y)$. Now, for $g \neq 0$, we show that there exists a $u \rightarrow v \rightarrow u$ walk of any given voltage $(g, h)$. There are $q(q-1)$ such pairs $(g, h)$. Notice that we achieve $q^{2}-q+1$ different voltages through $u \rightarrow v \rightarrow u$ walks consisting of two distinc arcs in $D_{q}^{*}$. Indeed let $W_{1}$ be a $u \rightarrow v \rightarrow u$ walk of net voltage $\left(i, i^{2}\right)+\left(j,-j^{2}-1\right)=\left(i+j, i^{2}-j^{2}-1\right)$ and let $W_{2}$ another $u \rightarrow v \rightarrow u$ walk of net voltage $\left(m+n, m^{2}-n^{2}-1\right)$. The equality $\left(i+j, i^{2}-j^{2}-1\right)=\left(m+n, m^{2}-n^{2}-1\right)$ implies either $i=m, j=n$ or $i+j=m+n=0$. Hence for any $\left(i, i^{2}\right)$ there exists just one voltage $\left(-i,-i^{2}-1\right)$ that yields $(0,-1)$. This means that the voltage $(0,-1)$ is achieved walking $q$ different $u \rightarrow v \rightarrow u$ paths, meanwhile there is just one $u \rightarrow v \rightarrow u$ walk with voltage $(g, h)$ for any $g \neq 0$. The arguments for walks from $v$ to $v$ are similar, using the set $X_{2}$.

(ii) Walks $W$ from $u$ to $v$ with net voltage $(g, h)$ : If $(g, h)=(0,0)$ the walk $W$ consists on the single arc $u \rightarrow v$ with voltage $\left(i, i^{2}\right)$, for $i=0$. If $g=0$ and $h \neq 0$, then the walk $W$ consists of the arc with voltage $(0,0)$ and a loop at vertex $u$ for $h \in X_{1}$ or at vertex $v$ for $h \in X_{2}$. Let $g \neq 0$, if $h=g^{2}$, there is a $u \rightarrow v$ walk $W$ of length one with net voltage $\left(g, g^{2}\right)$. If $h \neq g^{2}$ and $h-g^{2} \in X_{1}$ then $W$ is composed by the loop at vertex $u$ of voltage $\left(0, h-g^{2}\right)$ and the arc of the voltage $\left(g, g^{2}\right)$. If $h \neq g^{2}$ and $h-g^{2} \in X_{2}$ then we take first the arc with voltage $\left(g, g^{2}\right)$ and next the loop at vertex $v$ of voltage $\left(0, h-g^{2}\right)$.

(iii) Walks $W$ from $v$ to $u$ with net volgate $(g, h)$ : If $(g, h)=(0,-1)$ the walk $W$ consists on the single arc $v \rightarrow u$ with voltage $\left(i,-i^{2}-1\right)$, for $i=0$. If $g=0$ and $h \neq-1$, then $h+1 \neq 0$ and the walk $W$ consist of the 
arc with voltage $(0,-1)$ and a loop at vertex $u$ when $h+1 \in X_{1}$ or at vertex $v$ when $h+1 \in X_{2}$. Let $g \neq 0$, if $h=-g^{2}-1$, there is a $u \rightarrow v$ walk $W$ of length one with net voltage $\left(g,-g^{2}-1\right)$. If $h \neq-g^{2}-1$ and $h+g^{2}+1 \in X_{1}$ then $W$ is composed by the arc of the voltage $\left(g,-g^{2}-1\right)$ and the loop at vertex $u$ of voltage $\left(0, h+g^{2}+1\right)$. If $h \neq-g^{2}-1$ and $h+g^{2}+1 \in X_{2}$ then we take first the the loop at vertex $v$ of voltage $\left(0, h+g^{2}+1\right)$ and next the arc with voltage $\left(g,-g^{2}-1\right)$.

Corollary 3.2 For any prime power $q \equiv 1(\bmod 4)$ there is a family of mixed graphs of diameter 2 , undirected degree $r=\frac{q-1}{2}$ and directed out-degree $z=q$ approaching the Moore bound in the constant factor $\frac{8}{9}$ when $q$ tends to infinity.

Proof: The Moore bound $M(r, z, 2)$ for undirected degree $r=\frac{q-1}{2}$ and directed out-degree $z=q$ is $\frac{9}{4} q^{2}-\frac{1}{2} q+\frac{5}{4}$. Taking into account that $\left(D_{q}^{*}\right)^{\alpha_{1}}$ has order $2 q^{2}$, we have that

$$
\lim _{q \rightarrow \infty} \frac{\left|\left(D_{q}^{*}\right)^{\alpha_{1}}\right|}{M(r, z, 2)}=\frac{8}{9} .
$$

\section{References}

[1] Baskoro, E.T., L. Branković, M. Miller, J. Plesník, J. Ryan, J. Širáň, Large digraphs with small diameter: A voltage assignment approach, J. Comb. Math. Comb. Comput. 24 (1997), 161-176.

[2] D. Buset, M. El Amiri, G. Erskine, M. Miller, and H. Pérez-Rosés, A revised Moore bound for partially directed graphs, Discrete Math. 339 (2016), no. 8, 2066-2069.

[3] Gross, B. and R. Tucker. "Topological Graph Theory," Dover Publications, 2001.

[4] López, N., and H. Pérez-Rosés. Degree/diameter problem for mixed graphs, Procedia Computer Science 74 (2015), 2-9.

[5] McKay, B., M. Miller and J. Širáň, A note on large graphs of diameter two and given maximum degree, J Comb Theo Ser B 74 (1998), 110-118.

[6] Šiagiová, J., A note on the McKay-Miller-Širán̆ graphs, J Comb Th Ser B $\mathbf{8 1}$ (2001), 205-208. 